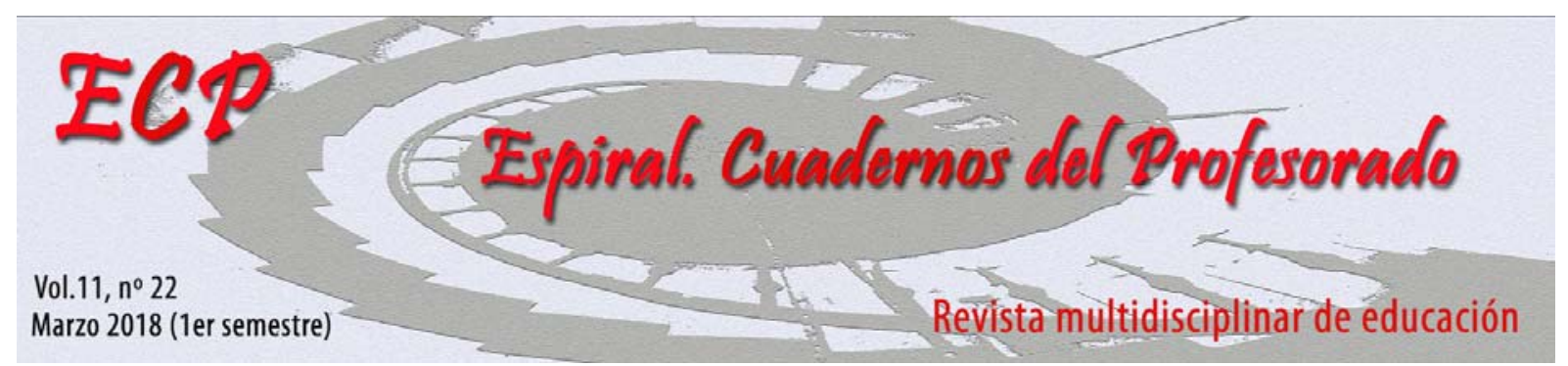

\title{
Actividades Físicas en el Medio Natural, Aprendizaje-Servicio y discapacidad intelectual
}

\section{Physical Activities in Natural Environment, Service-learning and Intellectual Disability

\author{
María Luisa Santos Pastor, L. Fernando Martínez Muñoz y Laura Cañadas
}

Departamento de Educación Física, Deporte y Motricidad Humana, Universidad Autónoma de Madrid, España

RESUMEN: Este artículo presenta el desarrollo de un proyecto basado en la metodología del AprendizajeServicio. Se aplicó en la asignatura Actividades Físico-Deportivas en el Medio Natural del Grado de Ciencias de la Actividad Física y el Deporte de la Universidad Autónoma de Madrid. El objetivo es mostrar las posibilidades formativas de esta metodología, donde la naturaleza y la práctica de actividad física son ejes vertebradores de la propuesta, además de generar un entorno inclusivo basado en el aprendizaje mutuo y recíproco entre estudiantes de la Universidad. El alumnado se forma en un contexto real, aprendiendo a plantear buenas prácticas en la naturaleza dentro de un programa compartido con jóvenes con discapacidad intelectual. Los resultados muestran los beneficios del uso de esta metodología, donde se aprenden los contenidos propios de las actividades físicas en el medio natural, fomentando un ocio activo-inclusivo en la naturaleza, además de generar un compromiso y una responsabilidad social.

Palabras clave: Aprendizaje-Servicio, actividades físicas en el medio natural, formación inicial, discapacidad intelectual.

\begin{abstract}
This research introduces the development of a project based on a Service-Learning methodology. It was applied to the course "Physical Activities in the Natural Environment" from the Physical Activity and Sport Sciences Degree at Autonomous University of Madrid. The aim of this research is to show the formative possibilities of this methodology where nature and physical activity are the main axes, in addition to generating an inclusive environment based on mutual and reciprocal learning among University undergraduates. The students are formed in a real context, learning to propose good practices in nature within a program shared with intellectually disabled young people. The results show the benefits of this methodology where students learn the contents of physical activities in the natural environment, promoting an active-inclusive leisure in nature while generating a commitment and a social responsibility.
\end{abstract}

Key words: Service-Learning, Physical activities in the natural environment, Initial training, Intellectual disability.

Santos Pastor, M. L., Martínez Muñoz, L. F., y Cañadas, L. (2018). Actividades Físicas en el Medio Natural, Aprendizaje-Servicio y discapacidad intelectual. Espiral. Cuadernos del Profesorado, 11(22), 52-60. 


\section{Introducción}

"La naturaleza educa al hombre para las relaciones sociales y por medio de las relaciones sociales". (J. Dewey)

La naturaleza se presenta como un espacio educativo con gran potencial pedagógico. A lo largo del tiempo ha habido diferentes corrientes educativas que han puesto en valor la educación al aire libre o en contacto con la naturaleza (v.g. la Institución Libre de Enseñanza-ILE), reclamándolo como principio básico para articular la educación de las personas mediante experiencias donde el medio natural surge como un espacio enriquecedor. Por su parte, Santos-Pastor (2000), expone las funciones que puede adquirir el espacio natural en los programas educativos, diferenciando entre: (1) eje temático, cuando es el tema que da forma a la acción educativa; (2) recurso pedagógico, cuando se emplea como instalación para la práctica dotando de sentido y significado a la acción; (3) eje pedagógico, en tanto que se está, se hace y se interacciona en y con el medio natural. Esta última función integra a las demás, al tiempo que justifica que la naturaleza sea un aula de aprendizaje donde dar sentido y significado a un proyecto educativo global.

Pese a que esta perspectiva contenga ciertos matices de utopía, ha sido considerada para su puesta en práctica en experiencias diversas. Podemos citar, entre otros, el modelo de la pedagogía de la experiencia de Hahn (Rose \& Sánchez, 2008) y su posterior desarrollo en el contexto americano de programas de aventura. Este programa incluía como pilar básico el servicio hacia los otros en la práctica de actividades en la naturaleza, fomentando la responsabilidad personal y social de los jóvenes, a la vez que una cohesión de grupo. La propuesta de pedagogía de la aventura de Parra (Parra \& Rovira, 2008) nos amplía esta línea de trabajo, mediante un proyecto cooperativo de humanización de los jóvenes a través de la realización de actividades en el medio natural, donde prima el desarrollo individual y social de los participantes y la educación en valores. En el momento actual, la tendencia de este tipo de propuestas toma como referencia la educación ambiental y el uso responsable de la naturaleza. En esta línea de trabajo, Freire (2011) presenta un modelo educativo más cercano a los ritmos y a la sencillez de la naturaleza, explorando las posibilidades que esta ofrece. El contacto con la naturaleza puede ayudar a fomentar la creatividad, el entusiasmo por el descubrimiento y el juego espontáneo; a adquirir capacidades para superar miedos y ganar confianza en uno mismo y en el entorno; y a alcanzar la conciencia del vínculo con el resto de seres vivos y con el ciclo de la vida.

Las continuas transformaciones de nuestra sociedad exigen reconsiderar las oportunidades para educar en la naturaleza, así como ser exploradas y descubiertas para la aplicación de experiencias educativas de éxito. La participación de todas las personas debe ser una clave fundamental para que todos los ciudadanos se puedan beneficiar de una educación en la naturaleza, al aire libre. La inclusión debe ser una condición del diseño de las prácticas educativas, considerando a todas las personas, con independencia de su procedencia, condiciones y posibilidades. Según Arnáiz (2005, p.65) "la inclusión implica preparar y apoyar a los profesores para que enseñen de forma interactiva. Los cambios en el currículo están estrechamente ligados a los cambios en su pedagogía".

Para afrontar este reto, el aprendizaje-servicio (ApS) es un método pedagógico activo, participativo y globalizador que nos permite hacer frente a propuestas inclusivas, al que varios autores definen a partir de términos como solidaridad, ayuda, apoyo a la comunidad o cooperación (GilGómez, Chiva-Bartoll \& Martí-Puig, 2015; Richards, Eberline, Padaruth \& Templin, 2015). El ApS incide sobre los contenidos curriculares a la vez que se presta un servicio a la sociedad, atendiendo una necesidad real no cubierta, desde una perspectiva de colaboración recíproca. Con su aplicación en la universidad es una clara apuesta por la formación de profesionales y ciudadanas y ciudadanos con compromiso social. La aplicación de este método en el contexto universitario propiciará en el alumnado un carácter de compromiso respecto a los colectivos con más necesidades de la comunidad donde se integran (Ostrander, 2004).

El ApS en Educación Física va a permitir al alumnado desarrollar una fuente de experiencias solidarias y de transformación social (Rubio, Campo, \& Sebastiani, 2014). Cuestión fundamental para abordar de forma inclusiva la acción docente en diferentes colectivos de personas con discapacidad 
utilizando como vehículo las actividades físico-deportivas en el medio natural (Luque-Valle \& Rico, 2012; Navarrete, 2009; Vía Libre, 2012).

Los estudios más recientes sobre Educación Física Inclusiva y ApS (Torrebadella-Flix, 2009; Tortosa, Caus, Vega, Blasco \& Rodríguez, 2011; Asún, 2014; Gil, Moliner, Chiva \& García, 2016) desvelan como el alumnado universitario es capaz de: mejorar su conciencia y responsabilidad social; comprender mejor las características del alumnado con discapacidad; interactuar socialmente con este colectivo y desterrar tópicos-prejuicios; trabajar en equipo para diseñar e intervenir en programas reales; obtener feedbacks inmediatos para mejorar sus propuestas; aumentar su motivación por la docencia; y obtener un alto grado de competencia profesional y personal en la formación. En cuanto al servicio ofrecido al colectivo de personas con discapacidad, podemos destacar como principal beneficio la inclusión en el ámbito universitario, además de re-descubrir la actividad física como medio para mejorar su calidad de vida y un modo de mejorar su socialización con sus iguales. En este marco, la propuesta que exponemos nos permite sensibilizar y mejorar las actitudes hacia las personas con discapacidad, posibilitando una verdadera inclusión a través de las actividades físico-deportivas en la naturaleza.

\section{Contexto de intervención}

El Proyecto que hemos desarrollado en la Universidad Autónoma de Madrid (UAM), se enmarca dentro de una metodología de Aprendizaje Servicio como propuesta formativa basada en el aprendizaje experiencial colaborativo entre la asignatura de Actividades Físico-Deportivas en el Medio Natural (AFDMN) del Grado de Ciencias de la Actividad Física y el Deporte (CAFYD) y el programa $^{1}$ cuyo plan de intervención se lleva a cabo desde la Fundación Prodis (http://www.fundacionprodis.org), institución sin ánimo de lucro, cuyo objetivo es formar a jóvenes con discapacidad intelectual para acceder al mercado de trabajo. Es la primera vez que esta experiencia se lleva a cabo dentro esta asignatura y de este Grado, y es la primera vez que esta colaboración (CAFYD-Promentor) a través de un proyecto ApS se produce. Su duración es de un cuatrimestre, la duración de la asignatura, actuando como experiencia piloto para su posible implantación en otras asignaturas de la titulación de CAFYD en años posteriores. Actualmente, desde la UAM se está dando impulso a este tipo de experiencias de ApS, aunque no hay ningún proyecto oficial en este sentido.

Se pretende que los estudiantes que cursan AFDMN, se formen desde la experiencia práctica en un contexto real de intervención en el ámbito de la discapacidad intelectual. Esta asignatura es de formación obligatoria, tiene 6 créditos ECTS y se desarrolla en el segundo semestre del segundo curso del Grado durante 16 semanas. Es impartida por dos profesores y otras personas de apoyo. Esta materia se compone de una parte teórica, desarrollada en el aula y otra práctica. Las salidas al entorno requieren la participación del alumnado, con la pretensión de que este adquiera cierta autonomía en el diseño y desarrollo de acciones prácticas.

El Programa Promentor surgió en el año 2004, por iniciativa de un grupo de personas vinculadas a la Universidad y a la Fundación Prodis, con el propósito de favorecer la inclusión de jóvenes universitarios con discapacidad intelectual. En el año 2009 se concedió una Cátedra de Patrocinio entre UAM y Prodis, lo que favorecería la continuidad de este proyecto, obteniendo un éxito reconocido tanto en el ámbito docente como en el de la investigación en relación al ámbito de la inclusión y de la discapacidad intelectual. En el marco de la Cátedra, se imparte un Título Propio denominado "Formación para la Inclusión Laboral", además de un curso de Formación Continua "Actualización y mejora en competencias socio-laborales" al que acceden los egresados del programa. Se distinguen dos grandes líneas de acción, que se concretan en (Izuzquiza \& Rodríguez, 2015): (1) favorecer la inclusión laboral de los alumnos a través del aprendizaje de competencias profesionales y (2) contribuir al desarrollo personal y social del alumnado, mediante una formación humanista que destaque el desarrollo de valores, la educación emocional o la adquisición de habilidades sociales.

${ }^{1}$ http://www.sindromedownvidaadulta.org/no19-febrero-2015/articulos-n19/programa-promentor-uam-prodis/ 


\section{Líneas de actuación del Proyecto Aprendizaje-Servicio}

El proyecto de ApS se orienta, principalmente, a impulsar el desarrollo personal y social del alumnado de CAFYD, futuro docente de Educación Física, mediante su participación e implicación activa en las prácticas de la asignatura de AFDMN, que son compartidas con otros estudiantes universitarios con discapacidad que participan en el programa Promentor de la Fundación Prodis.

\section{Propósitos del proyecto}

Este proyecto se dirige a mejorar el aprendizaje académico-curricular de las AFDMN, impulsando el desarrollo de una sociedad más justa, transformar la sociedad, favorecer el desarrollo de competencias profesionales a través de la experiencia solidaria y realizar un servicio a la comunidad teniendo como escenario la naturaleza. Tratamos de dar respuesta a un enfoque inclusivo, provocando la participación e interacción entre jóvenes universitarios de condiciones, posibilidades y necesidades diversas, a través de la práctica educativa en el medio natural. En este marco se ha creado un proyecto que requiere poner a prueba sus competencias docentes mediante el desarrollo de prácticas reales de AFDMN, junto con jóvenes universitarios con discapacidad intelectual. Los primeros podrán adquirir, experimentar y perfeccionar su capacidad como futuros docentes, tratando de diseñar e implementar acciones prácticas concretas en las que incluyan a personas con discapacidad, valorando las posibilidades de generar propuestas físico-recreativas en el medio natural, con carácter inclusivo y adaptado a las capacidades y condiciones de los posibles participantes. Los segundos, podrán participar en actividades físicas formativas-recreativas en el medio natural, cuyo enfoque se orientará a adquirir hábitos de práctica física en el medio natural para un ocio activo, así como vivenciar experiencias relacionadas con el medio natural, que les ayuden a concienciarse sobre su cuidado y respeto, al tiempo que mejorar su calidad de vida.

Los objetivos de aprendizaje de este proyecto son: (1) Descubrir las potencialidades educativas de las AFDMN en el marco de la discapacidad intelectual desde una metodología basada en el servicio a la comunidad; (2) conocer y practicar las AFDMN desde un enfoque integral, sostenible e inclusivo; y (3) adquirir una actitud de respeto hacia el medio natural mediante la práctica de AFDMN. Como objetivos de servicio a la comunidad, encontramos: (1) desarrollar actitudes inclusivas y de responsabilidad social hacia otros miembros de la comunidad educativa y la sociedad; y (2) fomentar la inclusión del alumnado con discapacidad en el contexto social de la universidad.

\section{Negociación de la intervención}

El punto de partida para el diseño del proyecto ha exigido dialogar con las partes implicadas en el mismo, tanto para valorar las posibilidades de la colaboración-servicio a emprender, como para conseguir la autorización pertinente para su desarrollo. La idea parte de los docentes de la materia de AFDMN con el objetivo de mejorar la formación de los estudiantes de CAFYD en aquellas

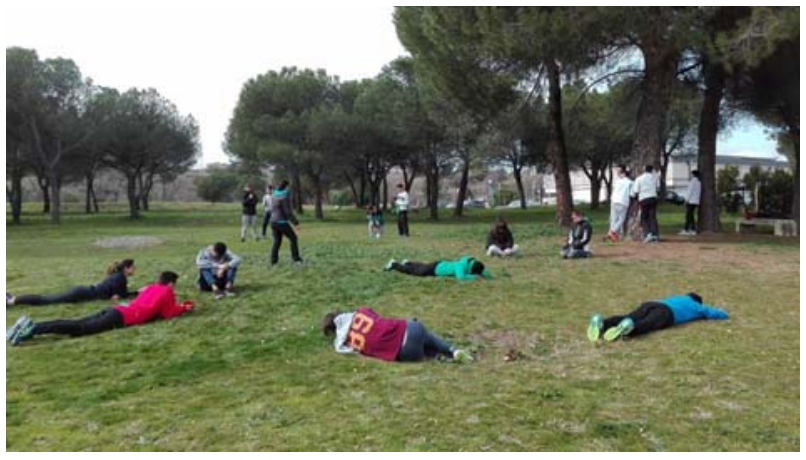

Figura 1. Descubriendo el medio natural. competencias relacionadas con la implementación de propuestas de actividades en el medio natural, a la vez que incidir en su formación para la inclusión y la responsabilidad social. Con este objetivo se contactó con los responsables del Programa PPro, y desde la materia de AFDMN se empezaron a implantar las diferentes propuestas que se recogen en este artículo.

Podemos distinguir, entre los docentes de la asignatura de CAFYD y la responsable del Programa PPro; así como otras personas que se involucran contractual o voluntariamente con su docencia (educadores-mediadores, colaboradores-docentes, becarias, voluntarios, etc.). También ha 
requerido informar a los Departamentos implicados ${ }^{2}$ y a la Facultad en la que se lleva a cabo la experiencia, tanto para tener su autorización formal, como para lograr la mayor cobertura e implicación de la comunidad educativa. Además, se ha informado y formado al alumnado de ambos programas, consiguiendo su aceptación para participar en las acciones previstas.

\section{Protagonistas y sus roles}

Los actores del proyecto son los estudiantes y los docentes, tanto del Grado de CAFYD como del Programa Promentor-Prodis (PPro), adquiriendo diferentes roles:

1) Profesorado: (a) Docentes del Programa ApS-AFMN, cuyas funciones son las de coordinar el proyecto, estableciendo los procedimientos necesarios para articular adecuadamente las distintas acciones previstas, en los tiempos y espacios determinados; supervisar y autorizar las propuestas que se diseñen y se pongan en práctica con los alumnos de PPro, poniendo en marcha las estrategias precisas para posibilitar un desarrollo de calidad. (b) Profesores asignatura AFDMN, que se encargan de diseñar las acciones teórico-prácticas que conforman el proyecto, su metodología y evaluación; además de establecer protocolos para la participación conjunta del alumnado de ambos programas, así como dinamizar el proyecto para poder desarrollarlo de manera óptima. (c) Profesores del Programa PPro, cuya misión es acompañar a los estudiantes con discapacidad intelectual, ofreciéndoles un apoyo adaptado para cada una de las actividades. (d) Colaboradores que apoyan el desarrollo de las prácticas previstas, ayudando a los profesores de la asignatura con la recogida de datos para la evaluación de la propuesta de ApS.

2) Estudiantes de ambos programas, participantes activos en las AFDMN, asumiendo diferentes roles y protagonismo. En el caso de los estudiantes de CAFYD (80 participantes), actúan como voluntarios-responsables de las actividades de PPro (Práctica Voluntaria), o como participantes acompañantes-apoyos del alumnado con discapacidad (25 participantes) cuando se incluyen en las actividades-salidas de AFDMN propuestas por la asignatura (Práctica Inclusiva).

\section{Contenidos del proyecto ApS-AFMN}

El proyecto ApS-AFMN se integra dentro de la asignatura como una opción o vía de aprendizaje a la que el alumnado puede optar de forma voluntaria. En él se incorporan exclusivamente los contenidos teórico-prácticos que se vinculan con las prácticas en el medio natural, dejando al margen aquellos que tienen un carácter más académico y técnico, por considerar que no es significativo para conseguir los propósitos previstos, aunque se harán en otros momentos-tiempos de manera paralela con los estudiantes del Grado de CAFYD, por formar parte de sus competencias profesionales, tal como se refleja en la guía docente.

Los contenidos a contemplar se dividen en función de su formato: teórico-práctico (talleres) y práctico (sesiones). Los primeros se desarrollan en un espacio concreto, por lo general cerrado, como el aula, en el contexto del campus universitario, cuyo objetivo se orienta a conocer los fundamentos teórico-prácticos de las AFDMN que se desarrollarán posteriormente en el medio natural. Los segundos, las prácticas físicas en el medio natural, se diferencian por los espacios en los que se llevan a cabo. Distinguimos entre: (1) entorno cercano (en el medio natural del propio campus universitario), (2) entorno próximo (en el medio natural cercano a la universidad -parque, bosque, monte-) y (3) entorno lejano (en el medio natural: parque nacional y otros espacios naturales).

\section{Metodología ApS-AFMN}

Las actuaciones del proyecto deben estar estructuradas y sistematizadas. Podemos diferenciar entre:

(1) Acciones iniciales, relacionadas con la información que los protagonistas reciben sobre el proyecto. Destacan reuniones iniciales y tutorías-seminarios con los grupos implicados. También se desarrollan procesos de formación específicos sobre la discapacidad intelectual. Resulta especialmente

\footnotetext{
2 Educación Física, Deporte y Motricidad Humana y Didáctica y Teoría de la Educación de la UAM (http://www.uam.es/ss/Satellite/FProfesorado/es/1234889757530/sinContenido/Departamentos.htm)
} 
significativa, por su valor formativo y pedagógico, la sesión de sensibilización que tiene lugar al iniciar la asignatura, en la que participan el alumnado de ambos programas (Grado y PPro). Se trata de un momento compartido entre el grupo, en el que cada participante expone sus dudas e intereses, además de lo que espera conseguir y aportar para el desarrollo del proyecto propuesto. La sesión de evaluación, tras acabar cada propuesta de intervención, es muy interesante y formativa ya que posibilita reconstruir y mejorar la práctica desarrollada.

Para complementar esta formación, se llevan a cabo diferentes actividades al comienzo del semestre, destacando: charlas temáticas sobre inclusión educativa del alumnado con discapacidad intelectual; talleres de AFDMN para aplicar metodologías favorecedoras de la inclusión de personas con discapacidad; prácticas simuladas en entornos naturales cercanos al campus, que sirven de laboratorio para reflexionar sobre las pautas didácticas, de gestión y organización de las salidas en el medio natural con personas con discapacidad.

(2) Acciones para el desarrollo del proyecto (tabla 1), que requieren la implicación y compromiso de los participantes (estudiantes-docentes-colaboradores). Distinguimos entre: práctica inclusiva, cuando los alumnos de PPro se incluyen en las clases prácticas de AFMN; y práctica voluntaria, cuando los alumnos del Grado de CAFYD, que voluntariamente lo seleccionen, imparten clase al alumnado de PPro. El principal objetivo es que se familiaricen con las AFDMN mediante su participación activa en las propuestas diseñadas por los estudiantes del Grado. Los contenidos de la intervención tienen un carácter mixto, integrando contenidos teórico-prácticos con su aplicación práctica. Entre ellos, destacan: senderismo por el Parque de Valdelatas, rallye fotográfico por el campus, acampada y juegos en el medio natural. Además del diseño y la intervención de la propuesta asignada, cada grupo confecciona el material audiovisual e impreso didáctico que sea necesario para el aprendizaje de los contenidos propuestos, adaptados al grupo y a sus particularidades.

Tabla 1.

Acciones del Proyecto ApS-AFMN.

\begin{tabular}{cc}
\hline $\begin{array}{c}\text { PRÁCTICAS VOLUNTARIAS } \\
\text { (*) Impartida por alumnos de CAFYD a los es- } \\
\text { tudiantes de Promentor }\end{array}$ & $\begin{array}{c}\text { PRÁCTICAS INCLUSIVAS } \\
(* *)\end{array}$ \\
\hline $\begin{array}{c}\text { A.1 Contenidos teórico-prácticos (Talleres) } \\
\text { A.1. Senderismo }\end{array}$ & $\begin{array}{c}\text { B. Contenidos prácticos } \\
\text { B.1. Entorno cercano }\end{array}$ \\
A.2. Orientación & EC1 Juegos en el medio natural \\
A.3. Escalada & EC2 Iniciación a la orientación \\
A.4. Acampada & EC3 Iniciación a la escalada \\
& B.2. Entorno próximo \\
A.2 Contenidos prácticos & EP1 Senderismo \\
Entorno cercano-próximo & EP2 Escalada \\
Juegos en el medio natural & EP3 Orientación con BTT \\
Senderismo por Parque de Valdelatas & B.3. Entorno lejano \\
Rally fotográfico & EL1 Raquetas1 \\
Acampada en el Campus & EL2 Raquetas 2 \\
& EL3 Senderismo 1 \\
& EL4 Raid + Escalada \\
& EL5 Senderismo 2 \\
& EL6 Piragüa + Trail \\
& EL7 BTT \\
& EL+1 Acampada \\
\end{tabular}

\footnotetext{
${ }^{3}$ Esta propuesta tiene por finalidad ofrecer a todos los alumnos/as de PPro conocimientos básicos teóricoprácticos de AFMN para facilitar una práctica más autónoma y segura cuando participen en las salidas al medio natural. Son impartidos por los estudiantes de CAFYD dentro de las acciones "Visita Voluntaria".
} 
Junto a estas acciones se realizan otras con carácter complementario, destinadas a organizar y gestionar los diferentes momentos de la intervención, entre los que destacan: reuniones de información inicial, organización general, tutorías, bien de manera individual, con cada uno de los grupos de trabajo o con todo el grupo de clase. Para conseguir el desarrollo de todas las tareas propuestas, este proyecto requiere de un trabajo colaborativo. Para ello, se articulan varios mecanismos que favorezcan la participación en las diferentes fases del proyecto (web, reuniones, twitter, etc.). El trabajo en red se convierte en un elemento de valor para conseguir nuestros propósitos.

\section{Evaluación}

Se propone una valoración continua de la intervención, recogiendo información de cada una de sus fases, apostando por una evaluación formativa. A medida que se vayan desarrollando los momentos del proyecto, se emplearán diferentes instrumentos: observaciones de las prácticas, diarios del alumnado, entrevistas (docentes, estudiantes) y reuniones de seguimiento que se desarrollan durante la puesta en marcha del proyecto. También se tiene previsto pasar a los estudiantes un cuestionario final, con el fin de poder analizar la adecuación e interés por la propuesta. Además, al alumnado se le solicita un diario de aprendizajes, mediante narraciones o grabaciones de audio, de sus experiencias en las actividades en las que participan.

\section{Primeros pasos. Valoración y reflexiones de la intervención con $\mathrm{ApS}^{4}$}

El contexto en el que se enmarca el proyecto es favorable para impulsar la consecución de los objetivos previstos en la programación. Los dos ámbitos de intervención (AFDMN + discapacidad intelectual) se sitúan en el mismo contexto universitario, lo que beneficia su aplicación y permite hacer una mejor gestión de las acciones programadas. En el marco de actuación más cercano (facultad-aulagrupo), nos surge la necesidad de informar e involucrar a todas las personas implicadas tanto en la gestión como en el desarrollo del proyecto. También se ha tenido que obtener permiso para implementar las acciones diseñadas, debiendo negociar con los protagonistas, estableciendo procesos de diálogo y acordando el procedimiento a seguir.

En la primera fase de su puesta en práctica hemos implementando algunas propuestas de AFDMN, tanto en espacios naturales cercanos como lejanos. Estas experiencias iniciales nos permiten valorar el interés que suscita este tipo de propuestas para el alumnado que participa. En el caso de los estudiantes de CAFYD, podemos concretar sus beneficios en varias líneas, destacando: (1) Compartir su formación con personas diversas, en concreto con el ámbito de la discapacidad intelectual en la naturaleza; (2) Ser una oportunidad única, real y necesaria para formarse, dotándose de herramientas teóricas y prácticas, aprendidas y experimentadas en condiciones reales en espacios naturales; (3) Ofrecer un servicio a la comunidad, mejorando la calidad de vida de las personas con las que trabajan; (4) Ejercer la responsabilidad social; (5) Adquirir y desarrollar sus competencias como docentes en el ámbito de la discapacidad intelectual y las AFDMN.

No podemos olvidarnos de los múltiples beneficios que reporta a los estudiantes de PPro incluirse en el programa de

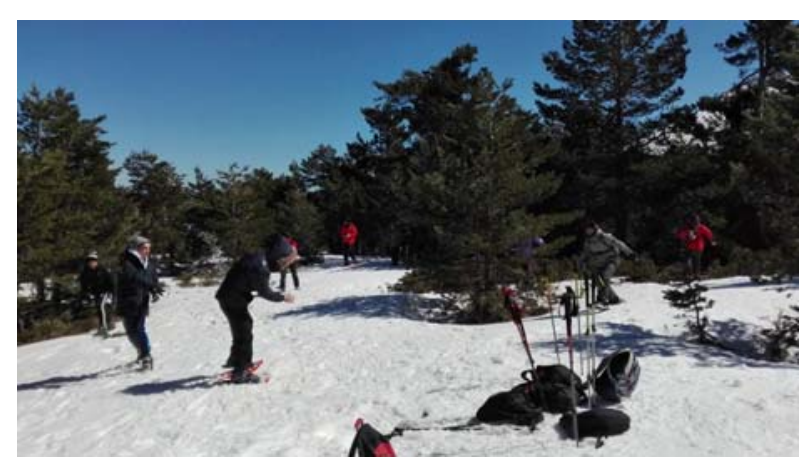

Figura 2. Aprendiendo en el medio natural. los estudiantes del Grado de CAFYD, compartiendo aprendizajes y experiencias en torno al medio natural como escenario pedagógico en el que se generan aprendizajes especiales y únicos. La

\footnotetext{
${ }^{4}$ En la actualidad este proyecto está en desarrollo, por lo que en estos momentos solo podemos ofrecer los resultados parciales de las acciones aplicadas.
} 
naturaleza les ofrece la oportunidad de verse sin diferencias, mejorando su calidad de vida desde un ocio activo en contacto con la naturaleza.

\section{Potencial inclusivo de las AFMN y formativo del ApS}

Las experiencias que hemos implementado hasta el momento, nos confirman el potencial inclusivo de las AFDMN y las posibilidades que el ApS tiene para la formación de los futuros profesionales de la actividad física y el deporte. La realización de este proyecto está teniendo un impacto formativo en el desarrollo profesional de los profesores implicados, pues nos ha permitido reflexionar profundamente sobre lo que puede aportar las AFDMN como práctica pedagógica para la inclusión de las personas, al tiempo que mejorar la formación de los profesionales de actividad físicadeportiva desde experiencias inclusivas, favoreciendo una sociedad más justa y un mundo más humano.

\section{Referencias}

Arnáiz, P. (2005). Atención a la Diversidad. Programa curricular. Costa Rica: Editorial Universidad Estatal a Distancia.

Asún, S. (2014). El aprendizaje de servicio en la Licenciatura en Ciencias de la Actividad Física y del Deporte de la Universidad de Zaragoza: una vía para potenciar la inclusión de las personas con discapacidad en la actividad física y el deporte. Barcelona: Paidotribo.

Freire, H. (2011). Educar en verde. Ideas para acercar a niños y niñas a la naturaleza. Barcelona: Graó.

Gil, J., Chiva, O., \& Martí, M. (2015). The impact of service learning on the training of pre-service teachers. Analysis from a physical education subject. European Physical Education Review, 21(4), 467-484. DOI: $10.1177 / 1356336 \mathrm{X} 15582358$

Gil, J., Moliner, O., Chiva, O., \& García, R. (2016). Una experiencia de aprendizaje-servicio en futuros docentes: desarrollo de la competencia social y ciudadana. Revista Complutense de Educación,27, 5373. DOI: $10.5209 /$ revRCED.2016.v27.n1.45071

Izuzquiza Gasset, D., \& Rodríguez Herrero, P. (2015). Programa Promentor (UAM-PRODIS). Un análisis de resultados de la primera experiencia de formación en España para personas con discapacidad intelectual en el ámbito universitario. Síndrome de Down: Vida Adulta, 19.

Luque-Valle, P., \& Rico, S. R. (2012). Las vías verdes son las instalaciones deportivas del futuro: espacios para realizar deporte en plena naturaleza. EmásF: revista digital de educación física, 19, 180-194.

Navarrete, J. (2009). Adaptación de senderos para personas con discapacidad: el derecho a la experiencia. Boletín de Interpretación, 21, 20-23.

Ostrander, S. A. (2004). Democracy, civic participation, and the university: A comparative study of civic engagement on five campuses. Nonprofit and Voluntary Sector Quarterly, 33, 74-93. DOI: $10.1177 / 0899764003260588$.

Parra, M. \& Rovira, C. M. (2008). Proyecto de humanización a través de la Pedagogía de la Aventura. AMEFIS Ceuta. I Congreso de Educación Física. Ceuta: Archivo General de Ceuta

Richards, K. A. R., Eberline, A. D., Padaruth, S., \& Templin, T. J. (2015). Experiential Learning Through a Physical Activity Program for Children With Disabilities. Journal of Teaching in Physical Education, 34(2), 165-188. DOI: 10.1123/jtpe.2014-0015

Rose, A., \& Sánchez, V. (2008). Kurt Hahn: precursor de las actividades en la naturaleza como elemento de la pedagogía de la experiencia. VI Congreso Internacional: El aula naturaleza. La actividad física en entornos naturales. Palencia: Patronato Municipal de Deportes.

Rubio, L., Campo, L., \& Sebastiani, E.M. (2014). Educación física y aprendizaje Servicio. Una combinación más que saludable. Tándem Didáctica de la Educación Física, 44, 7-14.

Santos-Pastor, M. L. (2000). Las actividades en el medio natural en la Educación Física Escolar. Tesis Doctoral. Universidad de Valladolid.

Torrebadella-Flix, X. (2009). Hacia un modelo de actividades físico deportivas inclusivas en el medio natural. EmásF, Revista Digital de Educación Física 23, 1-15.

Tortosa, J., Caus, N., Vega, L., Blasco, J., \& Rodríguez, C. (2011). El aprendizaje servicio como método de enseñanza en un curso de actividad física basada en el juego para personas con problemas de salud 
mental. En M. T. Tortosa, J. D. Álvarez, \& N. Pellín (coord.). IX Jornadas de Redes de Investigación en Docencia Universitaria: Diseño de buenas prácticas docentes en el contexto actual (pp. 653-668). Universidad de Alicante: Instituto de Ciencias de la Educación.

Vía Libre (2012). Buenas prácticas en desarrollo rural y personas con discapacidad. Madrid: Ministerio de Medio Ambiente y Medio Rural y Marino.

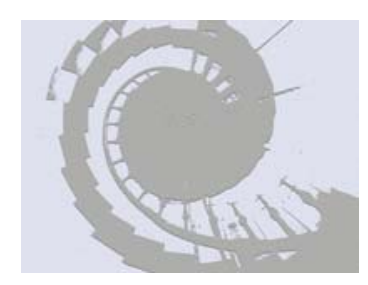

